\title{
Fatty acid dysregulation in the anterior cingulate cortex of depressed suicides with a history of child abuse
}

\author{
Kelly Perlman (D) ${ }^{1}$, Raphaël Chouinard-Watkins ${ }^{1,2}$, Arnaud Tanti ${ }^{1,3}$, Giulia Cisbani ${ }^{2}$, Massimiliano Orri (iD ${ }^{1}$, Gustavo Turecki (D) ${ }^{1,4}$, \\ Richard P. Bazinet ${ }^{2}$ and Naguib Mechawar (iD ${ }^{1,4}$
}

(c) The Author(s) 2021

Child abuse (CA) strongly increases the lifetime risk of suffering from major depression and predicts an unfavorable course for the illness. Severe CA has been associated with a specific dysregulation of oligodendrocyte function and thinner myelin sheaths in the human anterior cingulate cortex (ACC) white matter. Given that myelin is extremely lipid-rich, it is plausible that these findings may be accompanied by a disruption of the lipid profile that composes the myelin sheath. This is important to explore since the composition of fatty acids (FA) in myelin phospholipids can influence its stability, permeability, and compactness. Therefore, the objective of this study was to quantify and compare FA concentrations in postmortem ACC white matter in the choline glycerophospholipid pool (ChoGpl), a key myelin phospholipid pool, between adult depressed suicides with a history of CA (DS-CA) matched depressed suicides without CA (DS) and healthy non-psychiatric controls (CTRL). Total lipids were extracted from 101 subjects according to the Folch method and separated into respective classes using thin-layer chromatography. FA methyl esters from the ChoGpl fraction were quantified using gas chromatography. Our analysis revealed specific effects of CA in FAs from the arachidonic acid synthesis pathway, which was further validated with RNA-sequencing data. Furthermore, the concentration of most FAs was found to decrease with age. By extending the previous molecular level findings linking CA with altered myelination in the ACC, these results provide further insights regarding white matter alterations associated with early-life adversity.

Translational Psychiatry (2021)11:535; https://doi.org/10.1038/s41398-021-01657-4

\section{INTRODUCTION}

Child abuse (CA) is a major public health problem. In 2016, it was estimated that, worldwide, one billion children between 2 and 17 years of age experienced maltreatment in the previous year [1]. Overall, victims of CA present an increased burden of disease, both physical and psychological [2]. In particular, CA strongly increases the lifetime risk of suffering from major depressive disorder (MDD) and predicts an unfavorable course for the illness as well as poorer response to treatment [3]. Furthermore, CA comprises $54 \%$ of the population attributable risk (PAR) for depression and $67 \%$ of the PAR for suicide attempts [4]. Importantly, CA is the primary preventable risk factor in the development of mental illness [4]. It has been posited that the experience of CA modifies neural development such that the brain becomes more susceptible to the psychopathology of MDD and suicide [5], making it such a potent risk factor. Converging evidence in both animal and human studies suggests that the process of myelination contributes to this CA-induced vulnerability $[5,6]$.

Numerous CA-related myelin findings have been identified in the anterior cingulate cortex (ACC) a key limbic brain region involved in emotional regulation, processing of social pain, error detection, attention, cognitive control, reward-based decision making, and learning $[7,8]$. Our group has previously reported that in the ACC, there is an impairment in the myelin program of both the epigenome and the transcriptome, and that the myelin sheath around small-diameter axons is significantly thinner in postmortem brain samples from depressed suicides with a history of CA compared to matched samples from depressed suicides and non-psychiatric controls with no history of CA [9].

The myelin sheath is highly enriched in lipids, with proportions estimated at $70 \%$ to $85 \%$, in comparison to the typical membrane which is comprised of about $50 \%$ lipids [10]. Of the lipid pool, approximately $40 \%$ is constituted of phospholipids, of which nearly one-third is represented by the choline glycerophospholipid pool (ChoGpl) [11, 12]. It is estimated that of the ChoGpl pool, $90 \%$ is comprised of phosphatidylcholine (PC), with the remaining fraction being choline plasmalogens [13]. In phosphatidylcholine, a choline head group is esterified to the phosphate group at the Sn-3 position, and two fatty acids, usually one saturated (at the Sn-1 position) and one unsaturated (at the Sn-2 position), are attached to a glycerol backbone through ester linkages [14]. PC is a key structural and functional phospholipid in myelin and is a precursor for a number of cell signalling molecules [12].

Lipid studies of MDD have revealed that lipid dysregulation may be part of the pathophysiology of the illness and that it may be a promising target for treatment, often with a focus on plasma omega-3 FAs [15-17]. Several pre-clinical and postmortem human

\footnotetext{
${ }^{1}$ McGill Group for Suicide Studies, Douglas Mental Health University Institute, McGill University, Montreal, Québec, Canada. ${ }^{2}$ Department of Nutritional Sciences, Faculty of Medicine, University of Toronto, Toronto, Ontario, Canada. ${ }^{3}$ UMR 1253, iBrain, Université de Tours, Inserm, Tours, France. ${ }^{4}$ Department of Psychiatry, McGill University, Montréal,
}

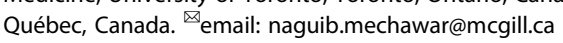


brain studies of MDD versus control individuals have identified differences in FA between groups, mostly when considering total lipids, though results are highly heterogeneous [18-23]. These inconclusive results may in part be explained by various factors in the experimental design of these studies. Notably, most studies in the field have examined total FAs, which can obfuscate any lipid class-specific FA findings. Moreover, there has often been a lack of information regarding the grey and white matter composition of the tissue samples, which is consequential because there are documented differences in FA composition between grey and white matter [24]. Lastly, most studies have reported brain FA content as a molar percent rather than absolute concentration, and the lack of an internal standard makes it impossible to control for the size of the FA pools.

The objective of this study was to quantify and compare ChoGpl FA concentrations in postmortem ACC white matter, between depressed suicides with a history of severe CA (DS-CA) compared to matched depressed suicides without CA (DS) and healthy controls (CTRL). Our analysis revealed significant differences in concentrations between groups in the FAs that compose the arachidonic acid synthesis pathway. Furthermore, the concentration of most FAs was found to be inversely correlated with age.

\section{METHODS \\ Samples}

This study was approved by the Douglas Mental Health University Institute's Research Ethics Board. Brain samples were provided by the Douglas-Bell Canada Brain Bank (DBCBB; http://douglasbrainbank.ca). All brains are donated to the Suicide section of the DBCBB by familial consent through the Quebec Coroner's Office. For each individual, the Quebec Coroner's Office determined the cause of death. Furthermore, a toxicology screen was conducted which ascertained the presence of any substances in the body at the time of death, as well as the substances taken during the 3 months prior to death.

All brains underwent a validated process known as psychological autopsy to retrieve phenotypic information [25]. The psychological autopsy was performed by trained professionals using existing documentation (coroner report, medical records, social service records) as well as standardized interviews with family members or informants best acquainted with the individual. These proxy-based interviews used adapted questionnaires for diagnosis of psychiatric disorder (e.g., SCID-I and SCID-II) and for ascertaining information on any childhood abuse (Childhood Experience of Care and Abuse [CECA]). Clinical vignettes are produced using all of the information collected, which is then reviewed by a panel of experts to confirm the diagnoses based on DSM-IV criteria.

For the CTRL group, suspicion of any psychiatric condition was an exclusion criterion. For both the DS or CTRL groups, any type of childhood abuse or neglect was an exclusion criterion. For all three groups, the presence or suspicion of any neurological or neurodegenerative disorder was an exclusion criterion. Individuals in the CTRL group died suddenly, generally in accidental deaths (e.g., work-related, car accident) or from a physical illness (e.g., cardiovascular arrest). All individuals in the DS-CA and DS groups died by way of suicide. As in previous work [9], there were strict criteria for inclusion in the DS-CA group that was selected for non-random severe abuse and/or neglect up until the age of 15 years. Specifically, the highest severity scores of 1 or 2 on either the sexual abuse, physical abuse, or parental neglect scales were required for inclusion.

As displayed in Table 1, frozen dorsal ACC white matter was obtained from a total of 101 individuals (15-85 years old) divided into three groups: male and female depressed suicides with (DS-CA; $n=39$ ) or without (DS; $n=36)$ a history of severe CA and matched sudden-death controls with no history of psychiatric nor neurological conditions (CTRL; $n=26)$. The sample size was determined based on both tissue availability and previous work from our group with the same subject groups [9]. Samples were dissected from coronal brain sections of the left hemisphere by expert brain bank staff, as described previously [9].

\section{Lipid extraction and FA quantification}

A minimum of $30 \mathrm{mg}$ of tissue was used per sample. Total lipids were extracted according to the Folch method (2:1 ratio of chloroform to methanol), homogenized in a glass douncer [26]. Lipids were separated into respective classes using thin-layer chromatography (TLC) on silica $\mathrm{H}$-plates. The mobile phase was 30:9:25:6:18 chloroform:methanol:2propanol:0.25\% KCl: triethylamine $(\mathrm{v} / \mathrm{v} / \mathrm{v} / \mathrm{v} / \mathrm{v})$. The plate was run until the solvent front reached $2 \mathrm{~cm}$ from the top. Once dried, the plate was sprayed with $0.1 \%(\mathrm{w} / \mathrm{v})$ 8-anilino-1-naphthalene sulfonic acid, and bands were viewed under ultraviolet light. The ChoGpl fraction was scraped off of the TLC plate and transmethylated using $14 \% \mathrm{BF} 3$ in methanol with incubation for $1 \mathrm{~h}$ at $100^{\circ} \mathrm{C}$. FA methyl esters from ChoGpl were quantified using a Varian 430 gas chromatograph (Bruker, Billerica, MA, USA). C17:0 was used as an internal standard and concentrations (measured in $\mu \mathrm{g} / \mathrm{g}$ ) of each FA were derived from the area under the curve (AUC) of its respective peak on the chromatogram. The relative percentage was calculated based on the individual and total FA concentrations. A graphical summary of this methodology is illustrated in Fig. 1.

\section{Gene expression}

The RNA sequencing data was previously generated in the Lutz, Tanti et al. (2017) study, which examined the transcriptome of ACC grey matter in DSCA versus CTRL groups only (for feasibility purposes). Briefly, RNA was exacted from frozen tissue, and only samples with an RNA integrity number (RIN) greater than 5 was used. Libraries were built using TrueSeq Standed Total RNA Sample Preparation and Ribo-Zero gold kits. All libraries were sequenced at the Genome Quebec Innovation Center on the Illumina HiSEquation 2000 platform, and each library with a sequencing depth of

Table 1. Subject information.

\begin{tabular}{|llll|}
\hline & CTRL & DS-CA & DS \\
\hline N total & 26 & 39 & 36 \\
\hline N female & 7 & 11 & 5 \\
\hline Age (yrs) & $46.46 \pm 19.86$ & $40.49 \pm 14.38$ & $50.19 \pm 11.34$ \\
\hline pH & $6.48 \pm 0.28$ & $6.50 \pm 0.34$ & $6.48 \pm 0.35$ \\
\hline PMI (h) & $42.30 \pm 27.70$ & $53.24 \pm 27.62$ & $57.04 \pm 28.43$ \\
\hline
\end{tabular}

Age, $\mathrm{pH}$, and PMI data are expressed as mean \pm standard deviation. To test for significant differences between groups for age and $\mathrm{pH}$, a one-way ANOVA was used with a cutoff of $p<0.05$. The data for PMI failed the Shapiro-Wilks test for normality, so a Kruskal-Wallis test was used with a cutoff of $p<0.05$. Age: $p=0.02, \mathrm{pH}: p=0.94$, PMl: $p=0.098$.
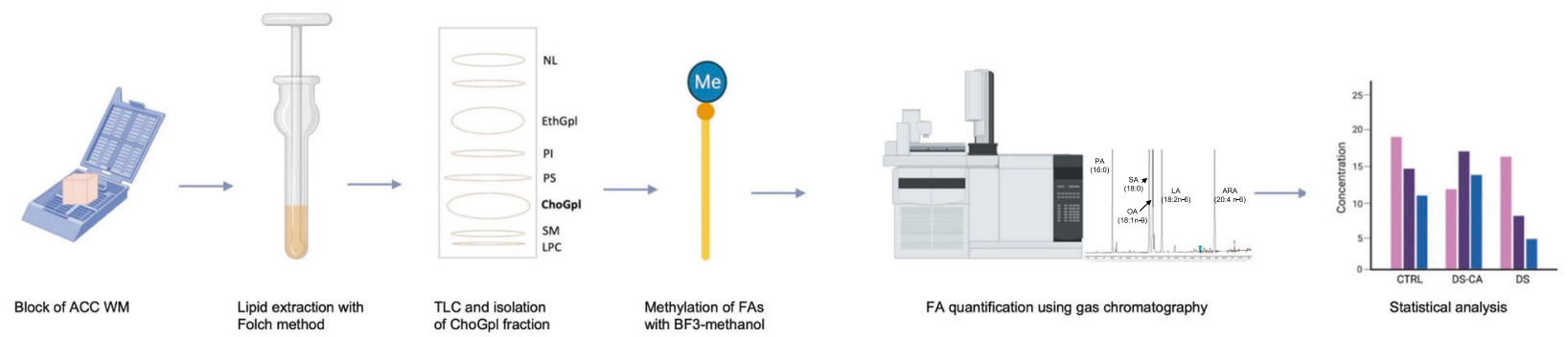

Fig. 1 Graphical overview of FA quantification methodology. NL: neutral lipids (e.g., cholesterol), EthGpl: ethanolamine glycerophospholipids, PI: phosphatidylinositol, PS: phosphatidylserine, ChoGpl: choline glycerophospholipids, SM: sphingomyelin, LPC: lysophosphatidylcholine. Figure was created using biorender.com. 


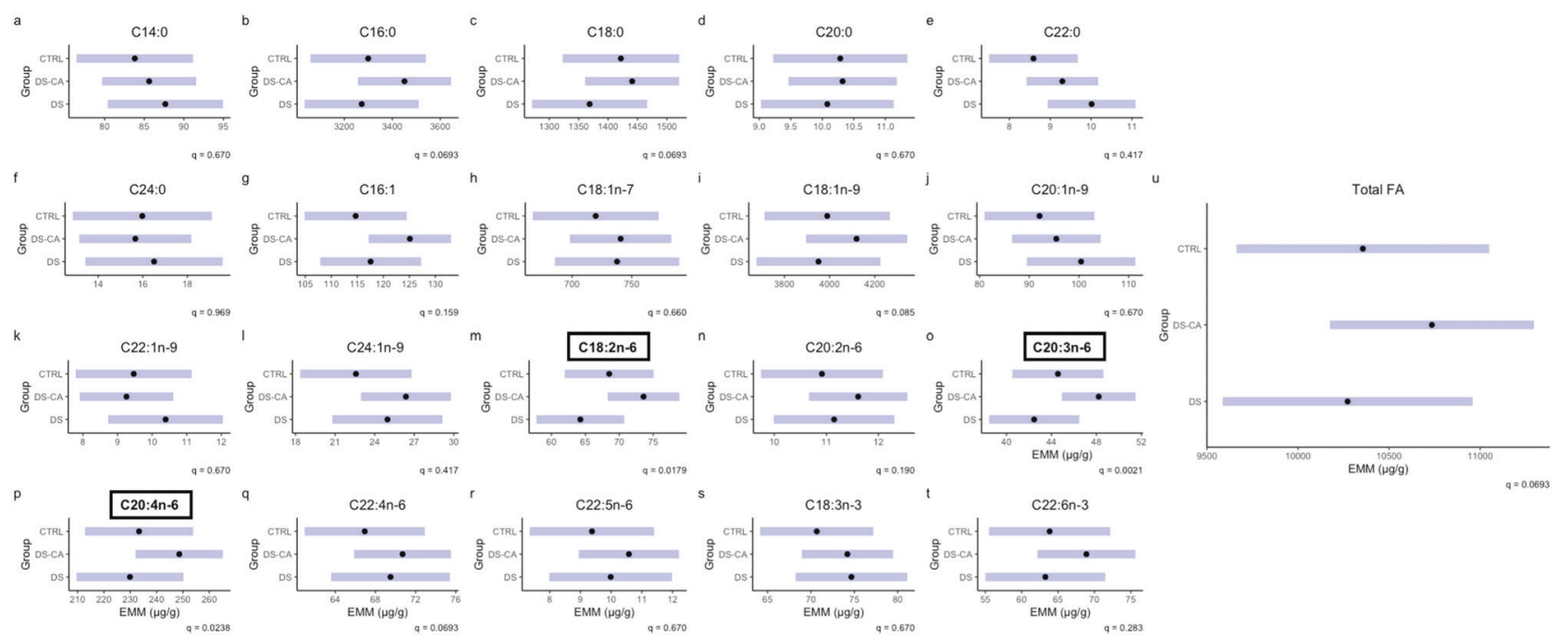

Fig. 2 Concentration of fatty acids in choline glycerophospholipids between groups. Black dots represent the estimated marginal means for the group and purple bars represent the $95 \%$ confidence intervals based on linear models for the concentration of FA as a function of the group with age, $\mathrm{PMI}, \mathrm{pH}$, and sex as covariates. Subplots with boxed titles in bold font indicate significance between groups with $q<0.05$. Concentrations are represented in $\mu \mathrm{g} / \mathrm{g}$.

approximately 62 million reads. Differential expression was calculated using the DESeq2 package [27].

\section{Statistics}

All statistics were computed in R. We modelled FA concentration and relative percentage as a function of the group with age, postmortem interval (PMI), $\mathrm{pH}$, and sex (encoded using a dummy variable) as covariates. Standard errors were computed using nonparametric bootstrap, $(k=$ 10,000 ) with the ANOVA.boot function of the Imboot package. Using this procedure, the coefficients of interest of the models were estimated 10,000 times in datasets using resampling, which generates an empirical distribution of coefficients from which robust standard errors can be estimated. This procedure achieves more robust and accurate estimations than parametric statistics in samples of limited size [28]. P-values were corrected for multiple comparisons using the Benjamini-Hochberg (BH) method, which yielded $q$-values $(q<0.05$ was considered significant). Group-by-age interactions were tested but none reached statistical significance after $\mathrm{BH}$ correction and as such were not included in the models. Estimated marginal means for groups were calculated across all covariates and plotted with the emmeans package. Pearson correlation was used to assess the relationship between FA concentrations and were considered significant at $p<0.05$.

\section{RESULTS}

\section{Sample exclusions}

Concentration and relative percentage data were generated with samples from 101 subjects (Table 1). One DS sample was excluded due to technical artifacts. As can be seen on the principal component analysis plot for concentration (Supplementary Figure 1), this sample is separated from all others across PC1 (i.e., the axis corresponding to the linear transformation that accounts for the most variance in the data), which clearly makes it an extreme outlier. We were not able to rerun this sample due to tissue unavailability. Furthermore, one CTRL sample was excluded from the between-group analysis because medical reports obtained later noted that this subject had been prescribed antidepressants, though no psychiatric diagnosis was noted.

\section{Between group modelling}

We compared 20 FAs between groups, as well as a total FA quantification for the concentration metric. FAs with concentrations significantly different between groups after $\mathrm{BH}$ correction were: $C 18: 2 n-6, C 20: 3 n-6, C 20: 4 n-6,(2 m, 20,2 p)$. Our analyses were not sufficiently powered to detect significant differences between individual pairs of groups in post-hoc tests. However, the estimated marginal means (EMM) for groups displayed the highest concentration in the DS-CA group for all significant FAs. There did appear to be a trend, in which the EMMs several of the FAs (including the total FA metric) were higher in the DS-CA group, though this is solely descriptive (Fig. 2). For each model with a significant group term, we calculated the percentage of variance explained by each significant factor (Supplementary Table 2).

When considering the group differences between FAs in relative percentage, we found that no FA reached significance after correction with $\mathrm{BH}$ (Fig. 3), despite highly similar patterns for $C 18: 2 n-6, C 20: 3 n-6, C 20: 4 n-6$, as in concentration. For reference, Supplementary Table 1 lists the relative percentage of individual FAs in ascending order.

\section{Age correlations}

Upon examining the relationships between FA quantities and the covariates of interest, we observed that age was the covariate most strongly correlated (in terms of the magnitude of the correlation coefficient) compared to the others, in particular when considering concentration (Supplementary Fig. 2A). Given the wide age span of the subjects included in this study, we were able to study the association between age and FA levels from adolescence through old age. Since group differences were not being considered here, we added back the CTRL subject excluded due to medication history.

Pearson correlations demonstrated significant relationships for 14 out of 20 FAs (plus the total FA metric) such that age was inversely correlated with the concentration of these FAs (Fig. 4a). The FAs with the highest correlation coefficient were C20:3n- 6 $(r=-0.44), 20: 4 n-6(r=-0.48)$ and C22:4n-6, $(r=-0.57)$. Only these FAs with the strongest negative correlations for concentration appeared to be reflected as decreased across the age span for relative percentage (Fig. 4b). Furthermore, none of the 4 FAs that were significantly directly correlated with age in terms of relative percentage (C16:1, C18:1n-7, C18:2n-6, C18:3n-3) reached significance when correlating age and concentration (Fig. 4b). As such, it is likely that these FAs may contribute a greater proportion of the total FA pool later in life as their concentrations remain fairly constant while others are decreasing. 

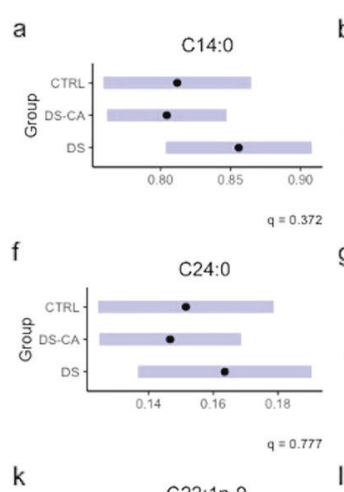

k
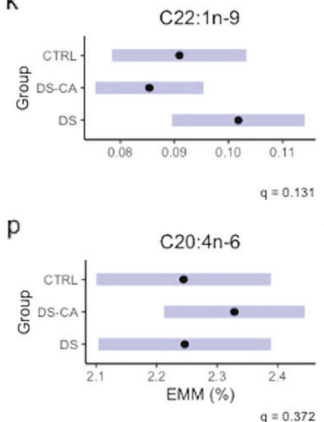

b

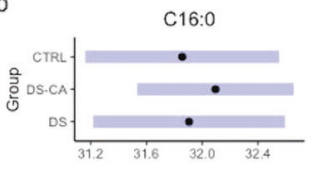

g
C

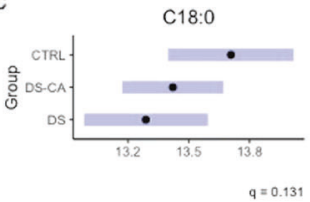

h

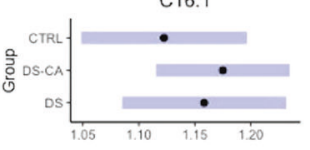

I

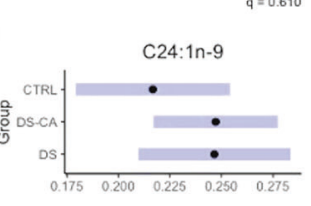

q

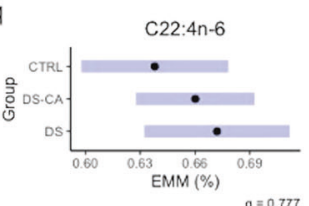

d

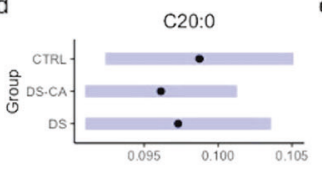

e

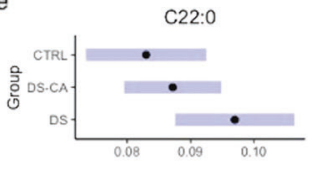

i

\section{西}

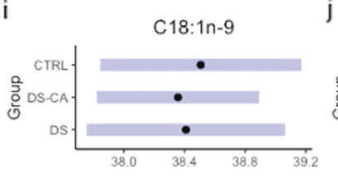

j

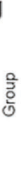

m $q=0.131$

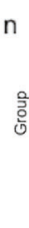

n

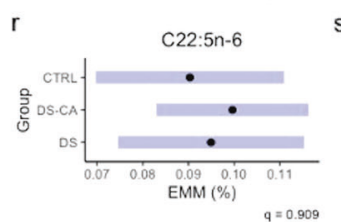

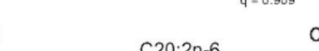

0
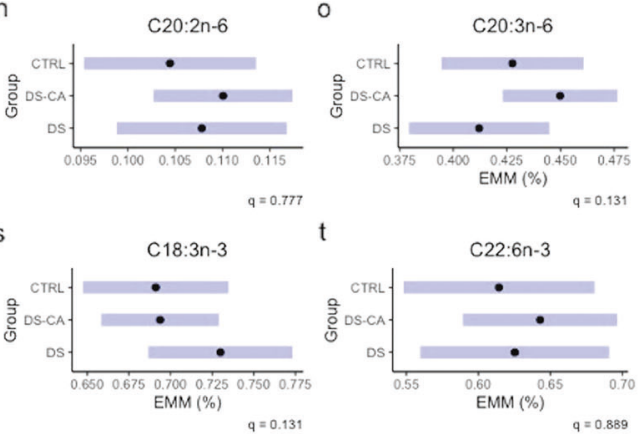

Fig. 3 Relative percentage of fatty acids in choline glycerophospholipids between groups. Black dots represent the estimated marginal means for group and the purple bars represent the $95 \%$ confidence intervals based on linear models for the relative percentage of FA as a function of the group with Age, PMl, $\mathrm{pH}$, and sex as covariates. The group term did not reach significance in any relative percentage model after correction for multiple comparisons.

\section{Transcriptomics}

Given that the FAs that showed significant differences between groups were all involved in the arachidonic acid (AA) synthesis pathway, we aimed to assess whether this finding was reflected in the respective gene expression profiles. We obtained a list of genes in the AA metabolic process (GO:0019369) and looked for the intersection of those genes with the genes from the ACC RNAseq with a nominal $p$-value $<0.05$. Table 2 lists the gene names and symbols, along with the corresponding nominal p-values, fold changes and log fold changes. We observed that key genes involved in AA metabolism, including PLA2G4A and PTGS2 (also known as (COX-2), were significantly differentially expressed between DS-CA and CTRL groups in the ACC grey matter.

\section{DISCUSSION}

In this study of the quantification of ChoGpl FAs in human ACC, our key findings are that (1) the concentration of key FAs are dysregulated between DS-CA, DS, and CTRL groups; (2) the concentration of most FAs as well as the total FA concentration tends to decrease with age; and (3) concentration appears to be a more robust metric as compared to relative percentage for this type of research.

This FA dysregulation observed between groups may be related to our previous finding that the myelin sheath of small caliber axons in the ACC white matter are thinner in the DS-CA group [9]. The composition of FAs in myelin phospholipids has been demonstrated to influence its stability [10], permeability [29, 30], and compactness [31]. Therefore, one potential mechanism for the altered myelin sheath properties observed in CA $[9,32]$ could be a disruption of the lipid profile that composes the membrane, resulting in changes to properties such as membrane fluidity, which can lead to alterations in structural integrity with consequences on efficient information flow.

This is speculated because FAs with higher concentrations in the DS-CA group (at least descriptively) comprise the majority of
ChoGpl according to abundance (Fig. 2, Supplementary Table 1) and accordingly reflected in the total concentration (Fig. 2u), possibly pointing to a greater quantity of overall ChoGpl in this group. There is also transcriptomic evidence to support this view, since genes coding for key enzymes in PC synthesis such as choline kinase alpha and Choline/ethanolaminephosphotransferase 1 are differentially expressed between the DS-CA and CTRL groups (Supplementary Table 3). The mechanisms linking these FA changes to larger-scale morphology changes require further study, with consideration of the other lipid classes in myelin.

To our knowledge, this is the first human postmortem study to examine the potential impact of CA on brain FAs. In rats, it was demonstrated that a maternal separation paradigm induced a shift in the plasma PUFAs towards a more pro-inflammatory profile, with an increased concentration of $A A$ in the maternally separated group [33]. In humans, previous work has investigated the relationship between plasma PUFA levels in young adults, early life trauma, and depressive symptoms, which revealed that low omega-3 FA levels and a history of trauma are both associated with persistence of depressive symptoms, but not correlated to each other [34]. However, plasma and brain FA levels do not appear to be correlated [35], emphasizing the importance of human postmortem brain tissue with well-documented histories of maltreatment for obtaining a comprehensive picture of lipidrelated changes associated with early-life adversity.

The AA synthesis-related FA and gene expression findings suggest a possible dysregulation of inflammatory processes in the DS-CA group. While the directionality of these findings appears contradictory, it must be highlighted that the RNA-seq data were generated from grey matter while the FA results from white matter. It remains unclear, however, whether these genes are differentially regulated between cortical compartments. A more likely explanation of the apparent discrepancy between gene expression and FA levels may be that elevated levels of all the quantified FAs in the AA synthesis pathway may have driven compensatory changes in gene expression. Also, while AA is a precursor to inflammatory mediators, 
a
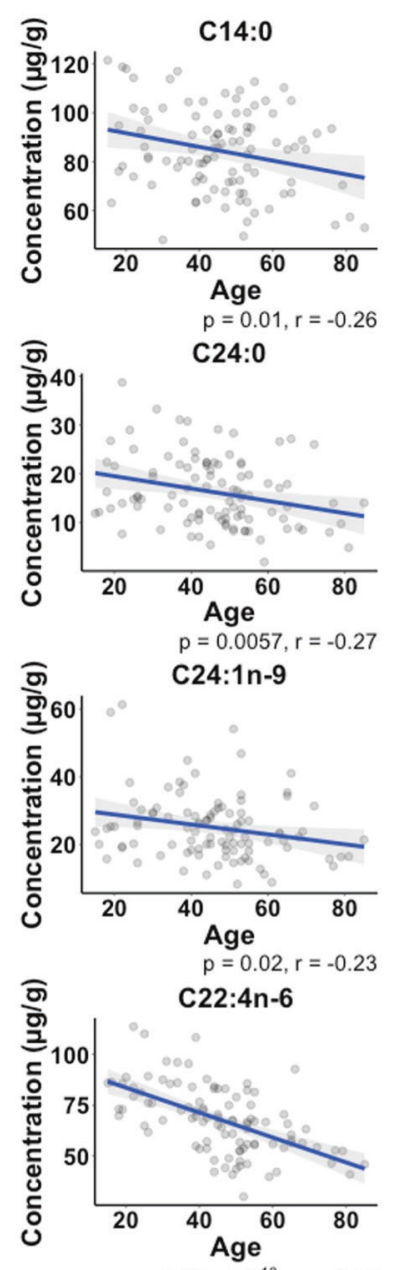

b
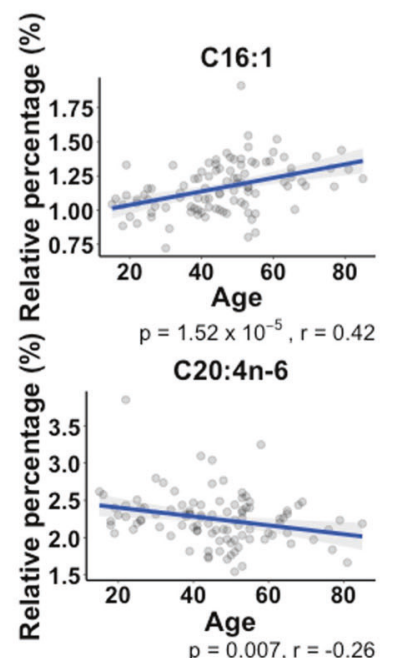

Concentration of fatty acids across age

C16:0

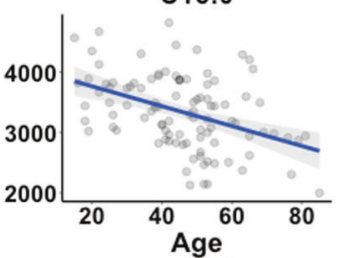

$p=1.45 \times 10^{-5}, r=-0.42$

C18:1n-7

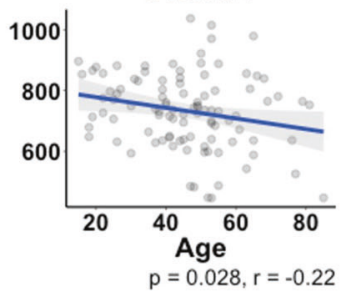

C20:2n-6

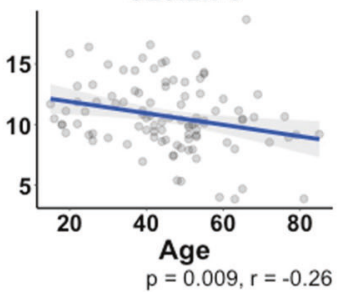

C22:5n-6

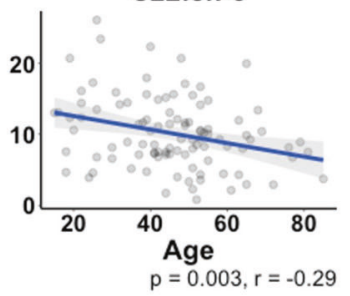

Relative percentage of fatty acids across age

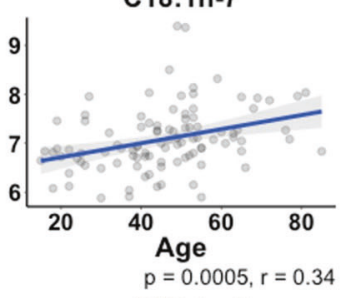

C22:4n-6

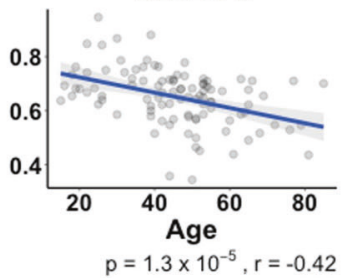

C18:0

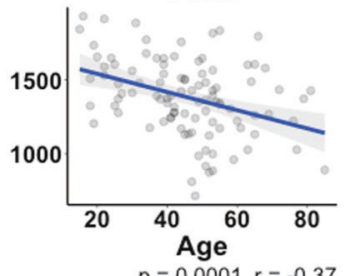

$p=0.0001, r=-0.37$

C18:1n-9

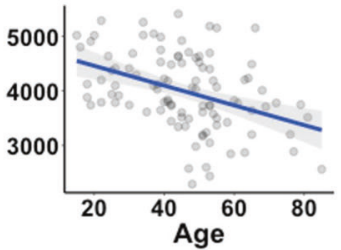

$p=3.46 \times 10^{-5}, r=-0.40$

C20:3n-6

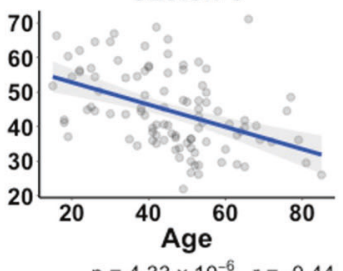

$p=4.33 \times 10^{-6}, r=-0.44$

C22:6n-3

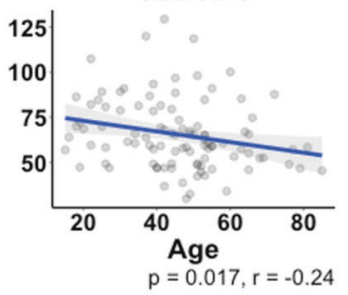

C18:2n-6
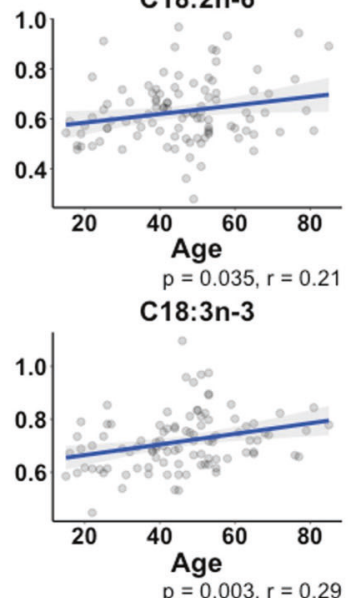

C20:0
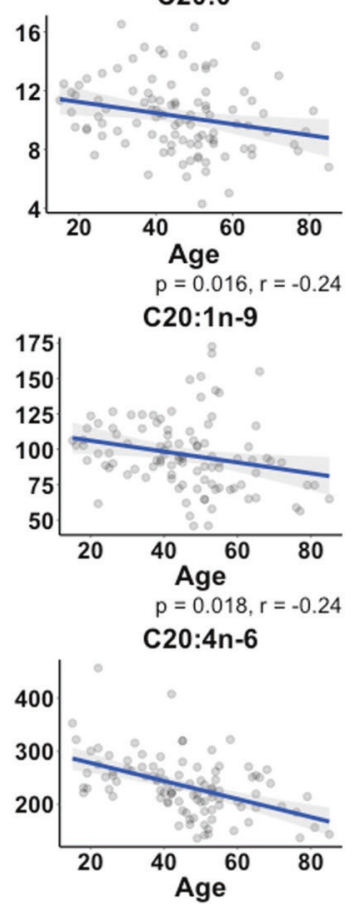

$p=4.59 \times 10^{-7}, r=-0.48$

Total FA

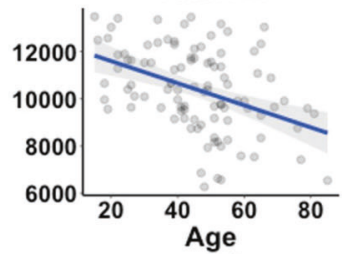

$p=2.00 \times 10^{-5}, r=-0.41$

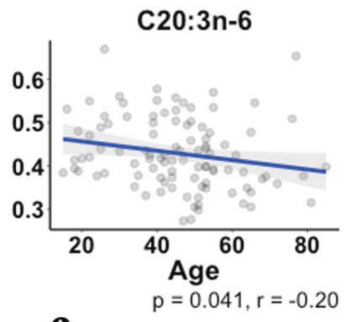

c

\begin{tabular}{|l|l|}
\hline \multicolumn{1}{|c|}{ Concentration } & \multicolumn{1}{c|}{ Relative percentage } \\
\hline$\downarrow \mathrm{C} 14: 0, \mathrm{C} 16: 0, \mathrm{C} 18: 0$, & \\
C20:0, C24:0, C18:1n- & $\downarrow \mathrm{C} 20: 3 n-6, \mathrm{C} 20: 4 n-6$, \\
$9, \mathrm{C} 20: 1 \mathrm{n}-9, \mathrm{C} 22: 1 \mathrm{n}-9$, & $\mathrm{C22:4n-6}$ \\
C20:2n-6, C20:3n-6, & $\uparrow \mathrm{C} 16: 1, \mathrm{C} 18: 1 \mathrm{n}-7$, \\
C20:4n-6, C22:4n-6, & $\mathrm{C} 18: 2 n-6, \mathrm{C} 18: 3 n-3$ \\
C22:5n-6, C22:6n-3, & \\
Total & \\
\hline
\end{tabular}

Fig. 4 Significant correlations between age and fatty acid quantitative metrics. Significant Pearson correlations between (a) age (years) and concentrations $(\mu \mathrm{g} / \mathrm{g})$ of FAs and $(\mathbf{b})$ age $(\mathrm{yrs})$ and relative percentage. $P$-values and $r$-values are listed under each subplot. A summary table of these significant correlations is presented in (c) in which a negative correlation is represented by a downward arrow and a positive correlation is represented by an upward arrow. 
Table 2. Genes involved in arachidonic acid metabolism with significant $p$-values.

\begin{tabular}{|c|c|c|c|c|}
\hline Gene symbol & Log fold change & Fold change & Nominal p-value & Gene name \\
\hline SPHK1 & -0.3391 & 0.7905 & 0.00091 & $\begin{array}{r}\text { sphingosine kinase 1 } \\
\text { prostaglandin-endoperoxide } \\
\text { synthase 2 }\end{array}$ \\
\hline PLA2G4 & -0.2441 & 0.8444 & 0.013 & $\begin{array}{c}\text { phoup } \\
\text { phospolipase A2, groum }\end{array}$ \\
\hline FADS1 & -0.2108 & 0.8640 & 0.023 & $\begin{array}{c}\text { IVAtosolic, calcium- } \\
\text { dependent }\end{array}$ \\
\hline CYP2U1 & -0.1935 & 0.8745 & 0.024 & fatty acid desaturase 1 \\
\hline GPX4 & -0.1104 & 0.9263 & 0.0087 & $\begin{array}{c}\text { cytochrome P450, family 2, } \\
\text { subfamily u, polypeptide 1 }\end{array}$ \\
\hline CYP2E1 & 0.1531 & 1.1120 & 0.0083 & glutathione peroxidase 4 \\
\hline & 0.2394 & 1.1805 & 0.0033 & $\begin{array}{c}\text { cytochrome P450, family 2, } \\
\text { subfamily e, polypeptide 1 }\end{array}$ \\
\hline
\end{tabular}

Purple colored rows represent decreased and green rows increased expression in DS-CA compared to CTRL samples.

it is implicated in other biological processes including ion channel regulation, cell signalling, neurotransmitter release, and even neurogenesis $[36,37]$, so the precise functional role it may play in this context requires further investigation. Furthermore, since dysregulation of the HPA axis is a consistent finding in individuals with a history of childhood maltreatment [5], it may be worth investigating the relationship between glucocorticoids as an inhibitor of phospholipase A2 (PLA2) [38] and any adaptive responses thereof.

Our findings concerning age are consistent with several previous postmortem studies demonstrating decreasing FA levels as a function of age [39-41]. In a study of total FAs from cerebral cortex homogenates, Carver and colleagues found decreasing concentrations of FAs, in particular omega-6 FAs, across ages 18 -88 years [40]. Hancock et al. found decreasing levels of $A A$ and adrenic acid (C22:4n-6) in combined phospholipids across ages 18 -104 years in both mitochondrial and microsomal membrane fractions of the hippocampus. Notably, AA and adrenic acid were the FAs with the largest negative correlation (according to $r$ value) in our data. Moreover, the administration of PUFAs has been shown to ameliorate cognitive deficits and even mild cognitive impairment that occurs in old age [42], which may reflect the beneficial effects of compensating for the observed loss over time.

This study also serves to illustrate that the concentration metric can be useful to prevent overlooking or mischaracterizing findings that may result from considering only a relative percentage. For example, a relative percentage cannot detect global upregulation or downregulation owing to the fact that it cannot control for the size of the FA pools. This observation has also been made in a study of mild cognitive impairment and Alzheimer's disease, such that no changes were observed when using a relative percentage metric but were identified using concentration data [35]. Absolute metrics obtained through the use of an internal standard should become common practice in the field of FA quantification in brain tissue.

This study is not without limitations. Firstly, we considered the ChoGpl pool as a whole, so we were unable to obtain a ratio of PC to choline plasmalogens for each subject. Despite the vast majority of the pool being comprised of PC, it is possible that the abundance ratio differed across subjects. This may be an especially important distinction given that plasmalogens have been documented to protect myelin against damage from reactive oxygen species [43], and oxidative stress is thought to be a mechanism linking early life stress to the development of later psychopathology [44]. Notably, we examined only the ChoGpl pool of phospholipids, and it was not possible to get a complete picture of any global dysregulation without studying other lipid classes. Furthermore, due to the low number of females in our sample, we were likely unable to reliably detect potential sex-specific differences. In addition, we extracted ChoGpl from blocks of ACC white matter, and therefore do not know if our findings are driven by the FAs of a specific subcellular compartment (e.g., the mitochondrial membrane). Another limitation comes from the fact that the available RNA-seq data only compared DS-CA and CTRL grey matter samples, so the differentially expressed genes could not be specifically associated with CA without further validation with white matter samples from all 3 groups. However, this transcriptomic data was used purely as a resource to explore potential links between FA differences and gene expression in relevant biological pathways. Finally, since we do not know the source of these FAs, we cannot comment on whether the group differences in FA concentration are reflective of alterations in synthesis, transport, and/or dietary intake. A future direction of this research is to probe dietary intake by assessing lipogenesis using carbon 13 isotopes in gas chromatographycombustion-isotope ratio mass spectrometry [45].

In summary, we have established that the concentrations of various ChoGpl FAs are altered between DS-CA, DS, and CTRL groups, and that they tend to decline across the lifespan. These findings warrant further investigation, in particular to establish the underlying mechanisms and to determine how they may contribute to the neurobiological vulnerability to psychopathology resulting from early-life adversity.

\section{REFERENCES}

1. Hillis S, Mercy J, Amobi A, Kress H. Global prevalence of past-year violence against children: a systematic review and minimum estimates. Pediatrics. 2016;137:2015-4079. peds

2. Dube SR, Felitti VJ, Dong M, Giles WH, Anda RF. The impact of adverse childhood experiences on health problems: evidence from four birth cohorts dating back to 1900. Preventive Med. 2003;37:268-77.

3. Lippard ETC, Nemeroff CB. The devastating clinical consequences of child abuse and neglect: increased disease vulnerability and poor treatment response in mood disorders. Am J Psychiatry. 2020;177:20-36.

4. Teicher MH, Samson JA, Anderson CM, Ohashi K. The effects of childhood maltreatment on brain structure, function and connectivity. Nat Rev Neurosci. 2016;17:652-66.

5. De Bellis MD, Zisk A. The biological effects of childhood trauma. Child Adolesc Psychiatr Clin North Am. 2014;23:185-222. vii 
6. Malter Cohen M, Jing D, Yang RR, Tottenham N, Lee FS, Casey BJ. Early-life stress has persistent effects on amygdala function and development in mice and humans. Proc Natl Acad Sci USA. 2013;110:18274-8.

7. Lavin C, Melis C, Mikulan E, Gelormini C, Huepe D, lbañez A. The anterior cingulate cortex: an integrative hub for human socially-driven interactions. Frontiers in Neuroscience 2013;7:64.

8. Giuliani NR, Drabant EM, Gross JJ. Anterior cingulate cortex volume and emotion regulation: is bigger better? Biol Psychol. 2011;86:379-82.

9. Lutz PE, Tanti A, Gasecka A, Barnett-Burns S, Kim JJ, Zhou Y, et al. Association of a history of child abuse with impaired myelination in the anterior cingulate cortex: convergent epigenetic, transcriptional, and morphological evidence. Am J Psychiatry. 2017;174:1185-94.

10. Williams KA, Deber CM. The structure and function of central nervous system myelin. Crit Rev Clin Lab Sci. 1993;30:29-64.

11. Devries G, Zetusky W, Zmachinski C, Calabrese V. Lipid composition of axolemma enriched fractions from human brain. J Lipid Res. 1981;22:208-16.

12. Poitelon Y, Kopec AM, Belin S. Myelin Fat Facts: An Overview of Lipids and Fatty Acid Metabolism. Cells 2020;9:812.

13. Chouinard-Watkins R, Chen CT, Metherel AH, Lacombe RJS, Thies F, Masoodi M et al. Phospholipid class-specific brain enrichment in response to lysophosphatidylcholine docosahexaenoic acid infusion. Biochimica et Biophysica Acta (BBA) - Mol Cell Biol Lipids. 2017;1862:1092-1098.

14. Hermansson M, Hokynar K, Somerharju P. Mechanisms of glycerophospholipid homeostasis in mammalian cells. Prog Lipid Res. 2011;50:240-257.

15. Walther A, Cannistraci CV, Simons K, Durán C, Gerl MJ, Wehrli S, et al. Lipidomics in major depressive disorder. Front Psychiatry. 2018;9:459.

16. Knowles EEM, Huynh K, Meikle PJ, Göring HHH, Olvera RL, Mathias SR, et al. The lipidome in major depressive disorder: Shared genetic influence for etherphosphatidylcholines, a plasma-based phenotype related to inflammation, and disease risk. Eur Psychiatry. 2017;43:44-50.

17. Liao Y, Xie B, Zhang H, He Q, Guo L, Subramanieapillai M, et al. Efficacy of omega3 PUFAs in depression: a meta-analysis. Transl Psychiatry. 2019;9:190.

18. McNamara RK, Hahn CG, Jandacek R, Rider T, Tso P, Stanford KE, et al. Selective deficits in the omega-3 fatty acid docosahexaenoic acid in the postmortem orbitofrontal cortex of patients with major depressive disorder. Biol Psychiatry. 2007;62:17-24.

19. McNamara RK, Jandacek R, Tso P, Dwivedi $Y$, Ren X, Pandey GN. Lower docosahexaenoic acid concentrations in the postmortem prefrontal cortex of adult depressed suicide victims compared with controls without cardiovascular disease. J Psychiatr Res. 2013;47:1187-1191.

20. Lalovic A, Levy E, Canetti L, Sequeira A, Montoudis A, Turecki G. Fatty acid composition in postmortem brains of people who completed suicide. J Psychiatry Neurosci. 2007;32:363-370.

21. Hamazaki K, Maekawa M, Toyota T, Dean B, Hamazaki T, Yoshikawa T. Fatty acid composition of the postmortem corpus callosum of patients with schizophrenia, bipolar disorder, or major depressive disorder. Eur Psychiatry. 2017;39:51-56.

22. Hamazaki K, Hamazaki T, Inadera H. Fatty acid composition in the postmortem amygdala of patients with schizophrenia, bipolar disorder, and major depressive disorder. J Psychiatr Res. 2012;46:1024-1028.

23. Tatebayashi Y, Nihonmatsu-Kikuchi N, Hayashi Y, Yu X, Soma M, Ikeda K Abnormal fatty acid composition in the frontopolar cortex of patients with affective disorders. Transl Psychiatry. 2012;2:e204-e204.

24. Skinner ER, Watt C, Besson JAO, Best PV. Differences in the fatty acid composition of the grey and white matter of different regions of the brains of patients with Alzheimer's disease and control subjects. Brain. 1993;116:717-725.

25. Dumais $A$, Lesage $A D$, Lalovic $A$, Séguin $M$, Tousignant $M$, Chawky $N$, et al. Is violent method of suicide a behavioral marker of lifetime aggression? Am J psychiatry. 2005;162:1375-1378.

26. Folch J, Lees M, Stanley GHS. A simple method for the isolation and purification of total lipides from animal tissues. J Biol Chem. 1957;226 1:497-509.

27. Love MI, Huber W, Anders S. Moderated estimation of fold change and dispersion for RNA-seq data with DESeq2. Genome Biol. 2014;15:550.

28. Efron B. Bootstrap Methods: Another Look at the Jackknife. Ann Stat. 1979;7:1-26

29. Chrast R, Saher G, Nave K-A, Verheijen MHG. Lipid metabolism in myelinating glial cells: lessons from human inherited disorders and mouse models. J Lipid Res. 2011;52:419-434

30. Manni MM, Tiberti ML, Pagnotta S, Barelli H, Gautier R, Antonny B. Acyl chain asymmetry and polyunsaturation of brain phospholipids facilitate membrane vesiculation without leakage. eLife. 2018;7:e34394.

31. Hoxha E, Balbo I, Parolisi B, Audano M, Montarolo F, Ravera F, et al. Elovl5 is required for proper action potential conduction along peripheral myelinated fibers. Glia 2021.

32. Forbes TA, Gallo V. All wrapped up: environmental effects on myelination. Trends Neurosci. 2017:40:572-587.
33. Clarke G, O'Mahony SM, Hennessy AA, Ross P, Stanton C, Cryan JF, et al. Chain reactions: early-life stress alters the metabolic profile of plasma polyunsaturated fatty acids in adulthood. Behavioural Brain Res. 2009;205:319-321.

34. Coryell WH, Langbehn DR, Norris AW, Yao JR, Dindo LN, Calarge CA. Polyunsaturated fatty acid composition and childhood adversity: Independent correlates of depressive symptom persistence. Psychiatry Res. 2017;256:305-311.

35. Cunnane SC, Schneider JA, Tangney C, Tremblay-Mercier J, Fortier M, Bennett DA, et al. Plasma and brain fatty acid profiles in mild cognitive impairment and Alzheimer's disease. J Alzheimers Dis. 2012;29:691-697.

36. Piomelli D, Astarita G, Rapaka R. A neuroscientist's guide to lipidomics. Nat Rev Neurosci. 2007;8:743-754.

37. Maekawa M, Takashima N, Matsumata M, Ikegami S, Kontani M, Hara Y, et al. Arachidonic acid drives postnatal neurogenesis and elicits a beneficial effect on prepulse inhibition, a biological trait of psychiatric illnesses. PLoS One. 2009;4:e5085-e5085.

38. Murakami M, Nakatani Y, Atsumi G, Inoue K, Kudo I. Regulatory functions of phospholipase A2. Crit Rev Immunol. 1997;17:225-283.

39. McNamara RK, Liu $Y$, Jandacek R, Rider $T$, Tso $P$. The aging human orbitofrontal cortex: decreasing polyunsaturated fatty acid composition and associated increases in lipogenic gene expression and stearoyl-CoA desaturase activity. Prostaglandins Leukot Ess Fat Acids. 2008;78:293-304.

40. Carver JD, Benford VJ, Han B, Cantor AB. The relationship between age and the fatty acid composition of cerebral cortex and erythrocytes in human subjects. Brain Res Bull. 2001;56:79-85.

41. Hancock SE, Friedrich MG, Mitchell TW, Truscott RJW, Else PL. Decreases in phospholipids containing adrenic and arachidonic acids occur in the human hippocampus over the adult lifespan. Lipids. 2015;50:861-872.

42. Janssen CIF, Kiliaan AJ. Long-chain polyunsaturated fatty acids (LCPUFA) from genesis to senescence: The influence of LCPUFA on neural development, aging, and neurodegeneration. Prog Lipid Res. 2014;53:1-17.

43. Luoma AM, Kuo F, Cakici O, Crowther MN, Denninger AR, Avila RL, et al. Plasmalogen phospholipids protect internodal myelin from oxidative damage. Free Radic Biol Med. 2015;84:296-310.

44. Schiavone S, Colaianna M, Curtis L. Impact of early life stress on the pathogenesis of mental disorders: relation to brain oxidative stress. Curr Pharm Des. 2015;21:1404-1412.

45. Meier-Augenstein W. Stable isotope analysis of fatty acids by gas chromatography-isotope ratio mass spectrometry. Analytica Chim Acta. 2002;465:63-79.

\section{ACKNOWLEDGEMENTS}

This work was supported by a CIHR Project Grant to NM (PJT-156346) and RPB. KP holds an FRQS scholarship and both GC and RCW hold CIHR postdoctoral fellowships. RPB hold the Canada Research Chair in Brain Lipid Metabolism. The Douglas-Bell Canada Brain Bank (DBCBB) is partly funded by a Healthy Brains for Healthy Lives (CFREF) and Brain Canada Platform Grants to GT and NM. The DBCBB is also funded by the Réseau Québécois sur le suicide, le troubles de l'humeur et les troubles associés (FRQS).

\section{AUTHOR CONTRIBUTIONS}

$\mathrm{NM}$, RPB, and KP conceptualized the study. RCW, AT, and GC conducted the experiments. $K P$ and $M O$ analyzed the data. $K P, R C W, A T, G C, R B P$, and NM interpreted the data. GT conducted the clinical characterization of the samples. KP wrote the paper with inputs from all authors.

\section{COMPETING INTERESTS}

RPB has received industrial grants, including those matched by the Canadian government, and/or travel support or consulting fees largely related to work on brain fatty acid metabolism from Arctic Nutrition, Bunge Ltd., DSM, Fonterra Inc, Mead Johnson, Natures Crops International, Nestec Inc. Pharmavite, and Sancero Inc. Moreover Dr. Bazinet is on the executive of the International Society for the Study of Fatty Acids and Lipids and held a meeting on behalf of Fatty Acids and Cell Signaling, both of which rely on corporate sponsorship. Dr. Bazinet has given expert testimony in relation to supplements and the brain. The other authors declare no competing interest.

\section{ADDITIONAL INFORMATION}

Supplementary information The online version contains supplementary material available at https://doi.org/10.1038/s41398-021-01657-4.

Correspondence and requests for materials should be addressed to Naguib Mechawar. 
8

Reprints and permission information is available at http://www.nature.com/ reprints

Publisher's note Springer Nature remains neutral with regard to jurisdictional claims in published maps and institutional affiliations. (c) (i) Open Access This article is licensed under a Creative Commons Attribution 4.0 International License, which permits use, sharing, adaptation, distribution and reproduction in any medium or format, as long as you give appropriate credit to the original author(s) and the source, provide a link to the Creative Commons license, and indicate if changes were made. The images or other third party material in this article are included in the article's Creative Commons license, unless indicated otherwise in a credit line to the material. If material is not included in the article's Creative Commons license and your intended use is not permitted by statutory regulation or exceeds the permitted use, you will need to obtain permission directly from the copyright holder. To view a copy of this license, visit http://creativecommons. org/licenses/by/4.0/.

(c) The Author(s) 2021 